Original Research Paper

\title{
Synchrotron Powder Diffraction Study of Radiation Damage in Langmuir Blodgett Nanotemplate Crystallised Protein
}

\author{
${ }^{1}$ Jonathan P. Wright, ${ }^{2}$ Eugenia Pechkova and ${ }^{3}$ Claudio Nicolini \\ ${ }^{I}$ ESRF, 71 Avenue des Martyrs, Grenoble, 38000, France \\ ${ }^{2}$ LNB-DIMES University of Genova \\ ${ }^{3}$ Nanoworld Institute, Fondazione Elba Nicolini, Bergamo, Italy
}

Article history

Received 2014-10-17

Revised 2014-11-06

Accepted 2014-11-07

Corresponding Author: Jonathan P. Wright,

ESRF, 71 Avenue des Martyrs, Grenoble, 38000, France

Email: wright@esrf.fr

\begin{abstract}
Polycrystalline samples of lysozyme were prepared with and without a Langmuir-Blodgett (LB) thin film template via both the hanging drop method and batch crystallisation. Powder diffraction methods are used to compare these samples and to measure their resistance to radiation damage at room temperature. The X-ray induced amorphisation of the samples was followed as a function of time and it was shown that diffraction does not entirely disappear even at very long exposure times. Two distinct kinetic timescales are evident suggesting that early and late stage processes are quite different. Radiation damage was also shown to be localized in the sample in the region where the beam impinges.
\end{abstract}

Keywords: Powder Diffraction, Lysozyme, Synchrotron Radiation

\section{Introduction}

Protein crystallization is the key bottleneck in the structure determination pipeline. New methods to produce crystalline proteins are therefore needed. By creating ordered 2D Langmuir Blodgett films of proteins, artificial $2 \mathrm{D}$ crystals can be produced and these can be used to seed protein crystallization (Pechkova and Nicolini, 2001; Pechkova et al., 2004). Previous experiments on templated crystals (Pechkova et al., 2009; 2010; Belmonte et al., 2012) have shown that the LB nanotemplated crystals show improved diffraction quality and greater resistance to radiation damage.

In any single crystal experiment, results which depend on the crystal quality are also sensitive to the selection and mounting of a specific crystal. The advent of powder diffraction methods which can be applied to protein samples (Von Dreele, 1999; Margiolaki and Wright, 2008) gives a new tool to examine crystalline quality. Powder methods measure data from millions of crystallites and so they are not so sensitive to outliers and instead give the bulk properties of a sample. The diffraction quality and resistance to radiation damage of different samples are compared using the powder diffraction method. We will show that radiation damage processes during the early stages of exposure to the X-ray beam are quite different to those at longer exposure time.

\section{Materials and Methods}

\section{Crystallisation}

Figure 1 shows the schematic method used for sample crystallization. Samples were prepared using a conventional hanging drop method and batch precipitation for comparison with the nanotemplate crystallised material. The nanotemplate crystallisation was performed as shown in (Pechkova and Nicolini, 2001; Pechkova et al., 2004).

For the samples prepared by the hanging drop method the crystals were harvested from the drops and gently crushed ready for powder diffraction analysis. Due to the size of the hanging drops the amount of crystalline material available from the drops was much smaller than for the batch prepared material.

Protein powder slurry was loaded into $0.8 \mathrm{~mm}$ diameter glass capillaries and centrifuged to pack the material into the end of the tubes. Excess liquid was removed from the capillary which was then sealed to prevent the sample from drying out. Capillaries were then fixed in a brass pin for mounting on the diffractometer.

\section{$X$-Ray Diffraction}

Powder X-ray diffraction data were collected at the Materials Science beamline, ID11, at the ESRF in Grenoble. An incident wavelength of $0.3444 \AA$ (36 
$\mathrm{keV}$ ) was selected using the Laue-Laue monochromator and the beam was focussed at the detector surface using the transfocator (Vaughan et al., 2011) with 46 Be lenses. The beam size at the sample position was defined by the X-ray source and focussing optics and was about 40 microns in the vertical and 60 microns in the horizontal.

2D diffraction data were recorded using a Frelon2K CCD camera (Labiche et al., 2007) and were integrated to give $1 \mathrm{D}$ diffraction patterns using the Fit2D software package (Hammersley et al., 1996). The sample to detector distance and beam centre were calibrated using a sample of Silver Behenate (Huang et al., 1993). Capillary tubes were rotated during data acquisition to ensure good powder averaging. The detector is positioned with the beamstop and direct beam position in the lower left corner of the image. This gives a better $\mathrm{Q}$ resolution for the same scattering range in comparison to the usual arrangement where the beam stop is at the centre of the image.

\section{Data Processing}

Integrated 1D diffraction profiles were analysed using the unit cell of tetragonal lysozyme (space group $\mathrm{P}_{3}{ }_{2} 2$, a $78, \mathrm{c} \sim 37$ Angstrom). The PRODD software (Wright, 2004) was used to carry out Pawley style intensity extractions to give unit cell and peak

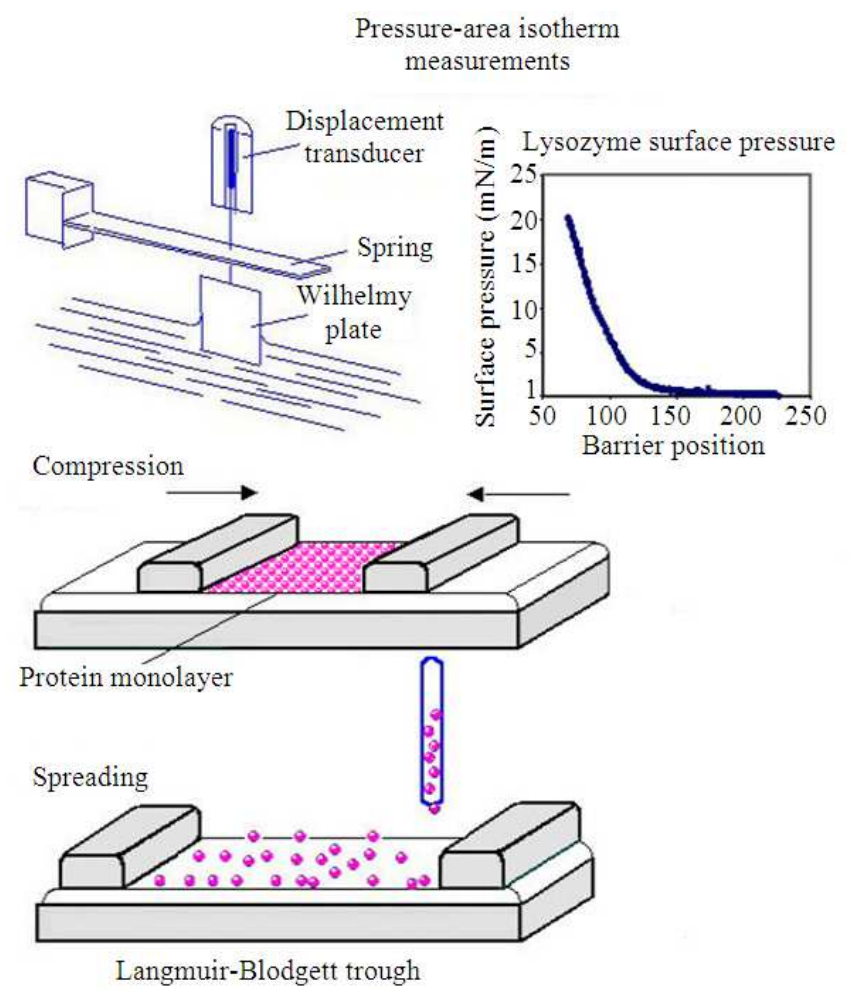

shape parameters for each diffraction pattern. The peak shape was well fitted with a pseudo-Voigt (Finger et al., 1994) function where the width as a function was parameterised as:

$$
\begin{aligned}
& \sigma^{2}=U \tan ^{2}(\theta)+V \tan (\theta)+W \\
& \Gamma=X / \cos (\theta)+Y \tan (\theta)
\end{aligned}
$$

$\Gamma$ and $\sigma$ are the widths of the Lorentzian and Gaussian components of the pseudo-voigt peak. After initial processing it was decided to fix the values of $U, V$ and $\mathrm{Y}$ to zero and refine only the $\mathrm{W}$ and $\mathrm{X}$ values.

To process the data as a function of time the integrated peak intensities were fixed at the initial values and a single overall scale factor was refined $($ FINT $=$ Fixed Intensity mode $)$. For each profile the unit cell parameters, scale, peak parameters (W and X) and background were refined. Typical refinements are shown on Fig. 4 for patterns 10 and 900 where the data are shown with and without subtraction of the background. In the early stages of the data processing the peaks are strong with respect to the background, but at a later stage the peaks are very weak. During the course of processing these data a new background function had to be introduced in the PRODD refinement code to allow refinement of a cubic spline interpolation of the background.

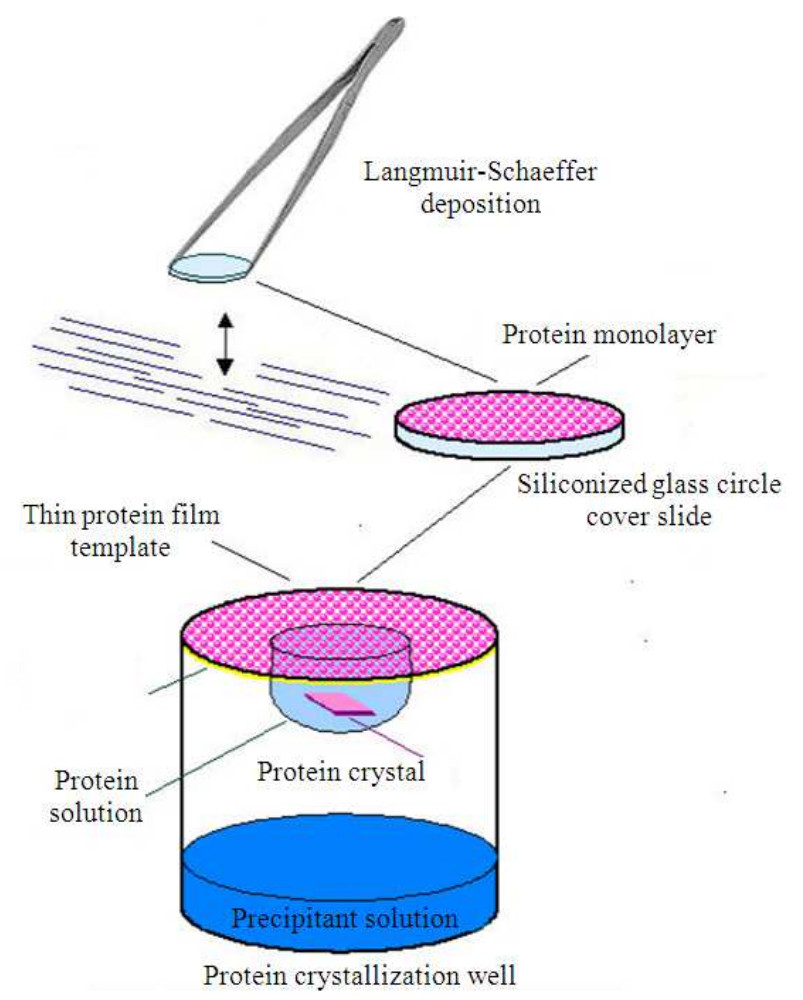

Fig. 1. Schematic showing the sample preparation. The protein monolayer is used to seed crystallisation 


\section{Results}

Four samples were studied, to compare conventional crystallisations with nanotemplated samples and also to compare the batch and hanging drop methods. Figure 2 compares the $2 \mathrm{D}$ images recorded for the 4 different samples in the initial exposures, prior to significant radiation damage. In the batch crystallisation there were a larger number of crystallites present and much stronger powder rings were observed. For the drop nano-templated sample there are weak powder rings and for the conventional crystallisation there are isolated diffraction spots. By using conventional drop crystallisation the crystals were too large for powder diffraction. Therefore the rest of the analysis focuses on the batch samples.

Figure 3 shows the integrated one dimensional profiles of the batch crystallised samples. In the upper panel the data from the LB templated sample is compared to the conventional batch crystallisation. In the inset it is clear that the LB sample has a slightly improved signal to background ratio and some very small peak shifts are evident. Note that the peak shifts are anisotropic, some peaks are displaced by more than others which is indicative of an anisotropic change of the unit cell parameters. In the lower panel of Fig. 3 the data are over-plotted as a function of image number (and therefore radiation dose). The loss of the diffracted intensity is accompanied by an increase in the background. This gives direct evidence that radiation exposure has converted crystalline protein into amorphous protein, which gives a smooth scattering curve, without sharp diffraction peaks. Even at very long exposure times, weak peaks can still be seen superimposed on the background.

The fits to the data to extract unit cell and peak shape parameters were generally very good and they are shown on Fig. 4. At long exposure times (lower row) the data are completely dominated by the background contribution and so this had to be very well fitted and carefully subtracted in order to leave only the peaks behind. In the right column of Fig. 4 the fits are shown with the background removed. After very long exposure times the signal to noise ratio is reduced, but clear peaks are still evident and these could be well fitted.

The numerical results of the sequential fitting are plotted on Fig. 5. In all four plots in Fig. 4 the behaviour of the samples is distinct in two time regions. During the early part of the experiment, up to image 40 or so, there are rapid changes and then later the system stabilises.

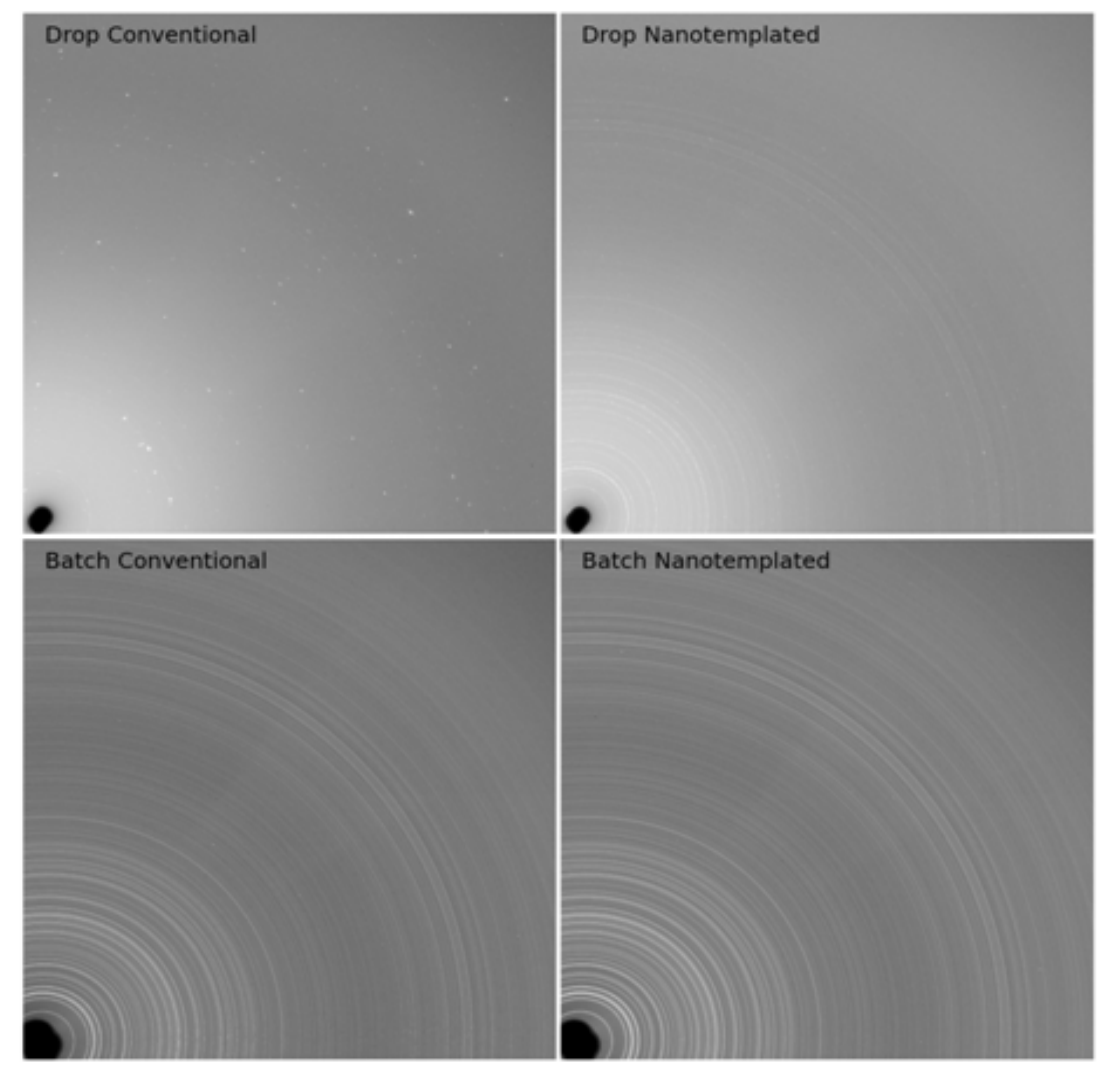

Fig. 2. 2D images from the Frelon CCD area detector at beamline ID11 (wavelength $0.3444 \AA$ ) for the four different samples in the initial state 

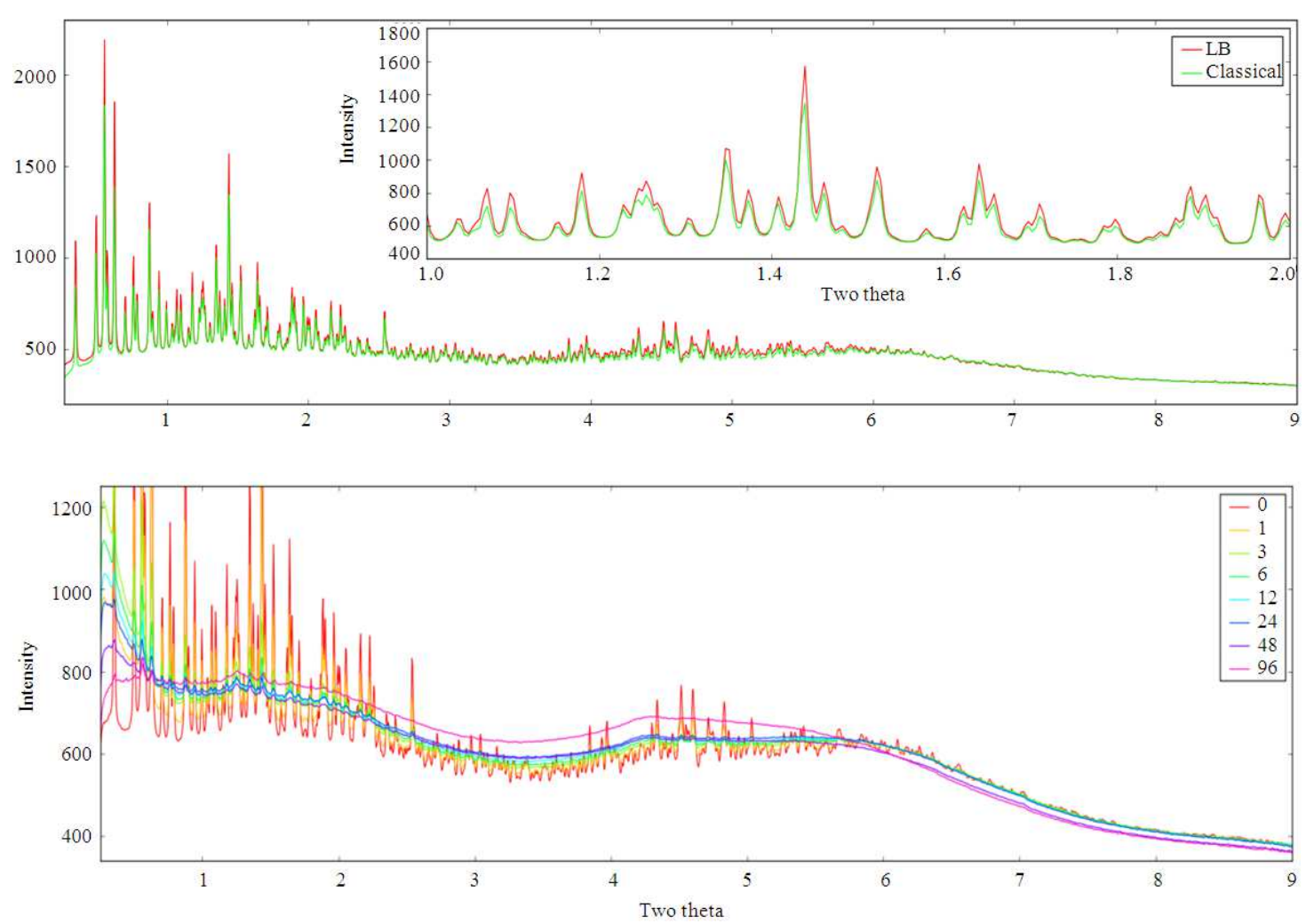

Fig. 3. Upper panel: Comparison of LB versus classical batch crystallisation. Lower panel: Evolution of the diffraction patterns with exposure time
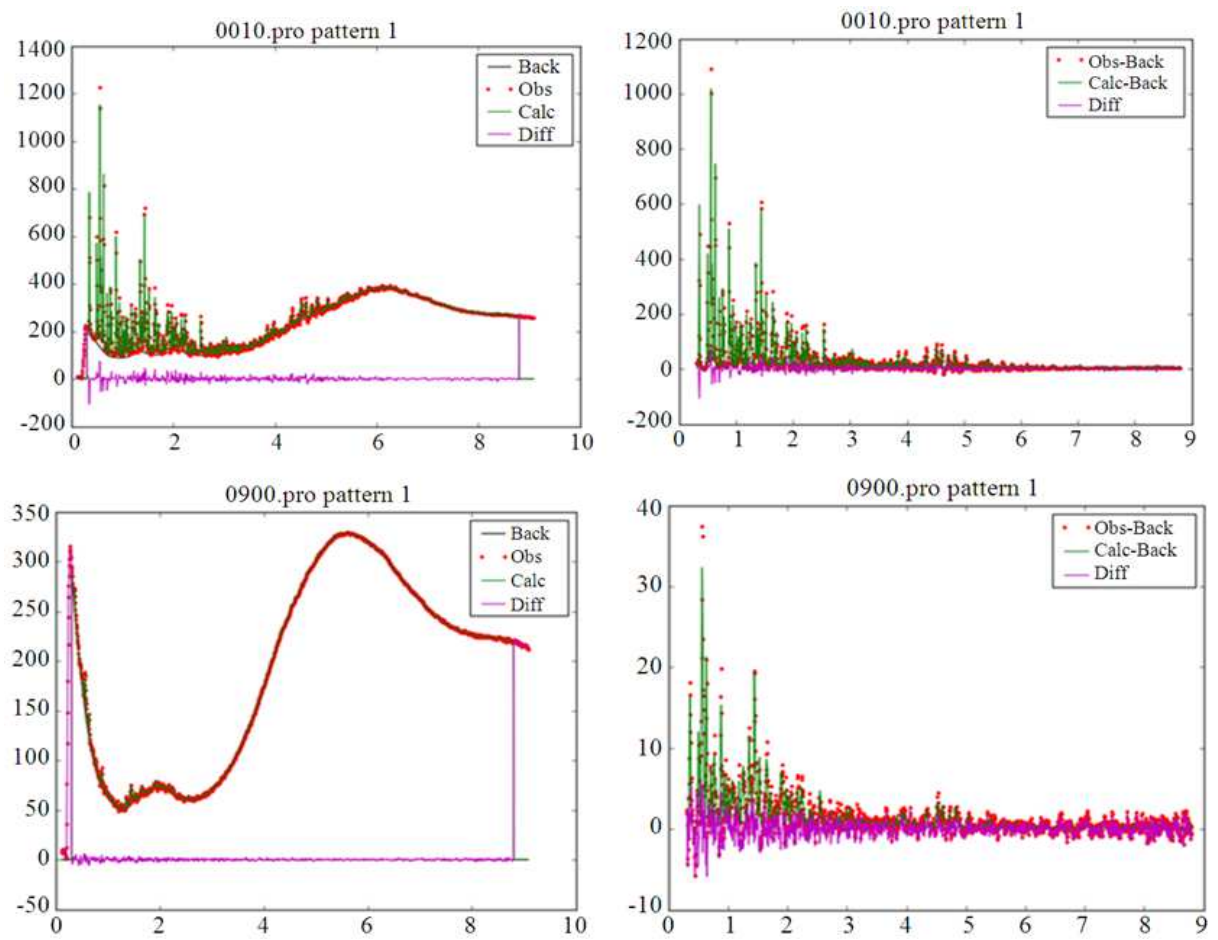

Fig. 4. Fits to the data to extract unit cell parameters and peak widths. Upper row: Image number 10, Lower row: Image number 900. Left column: Observed data and fit including the background. Right column: fit with background subtracted 

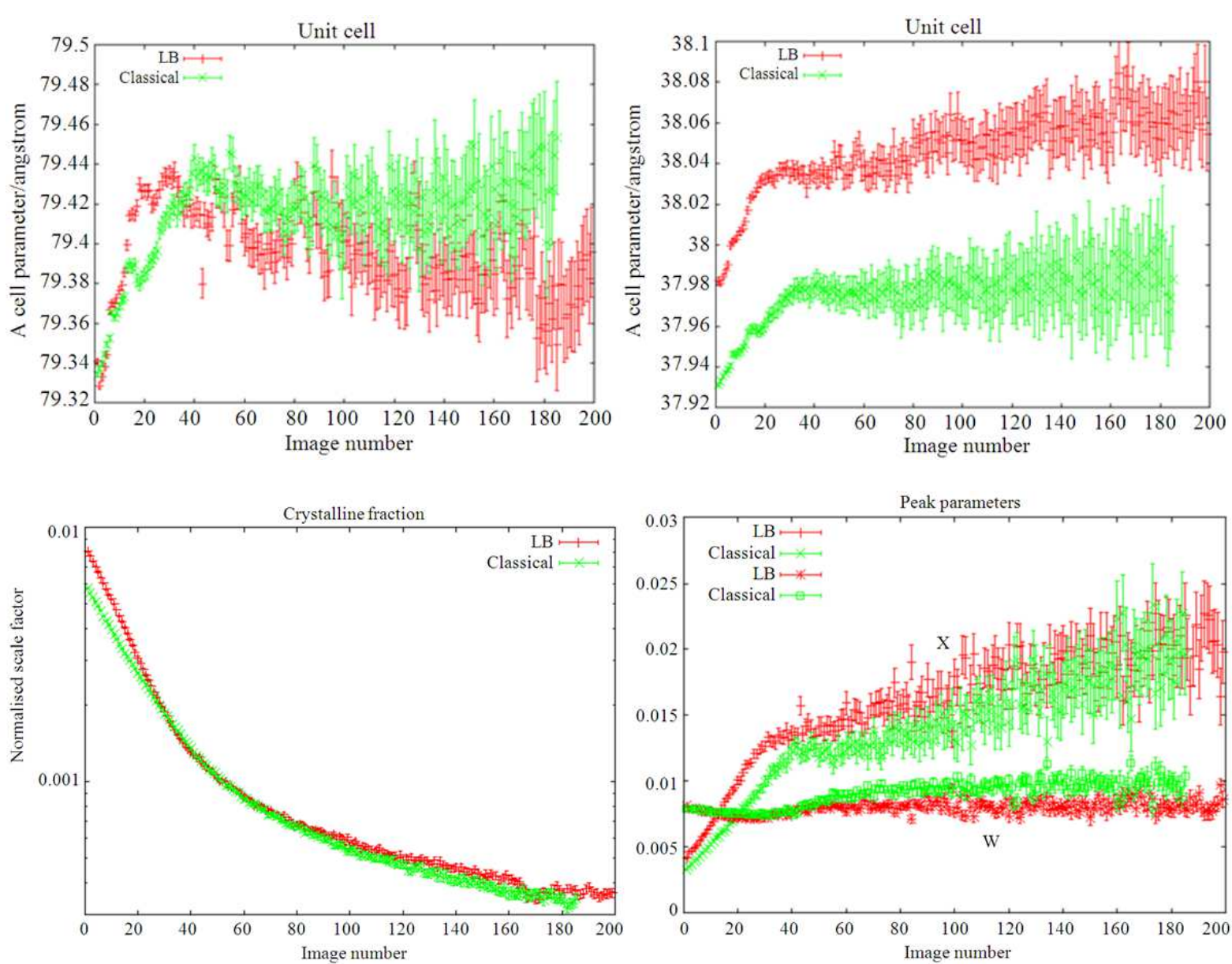

Fig. 5. Top left and right: Evolution of the 'a' and 'c' unit cell parameters. Bottom left: Evolution of the scale factor and bottom right, evolution of the peak shape parameters

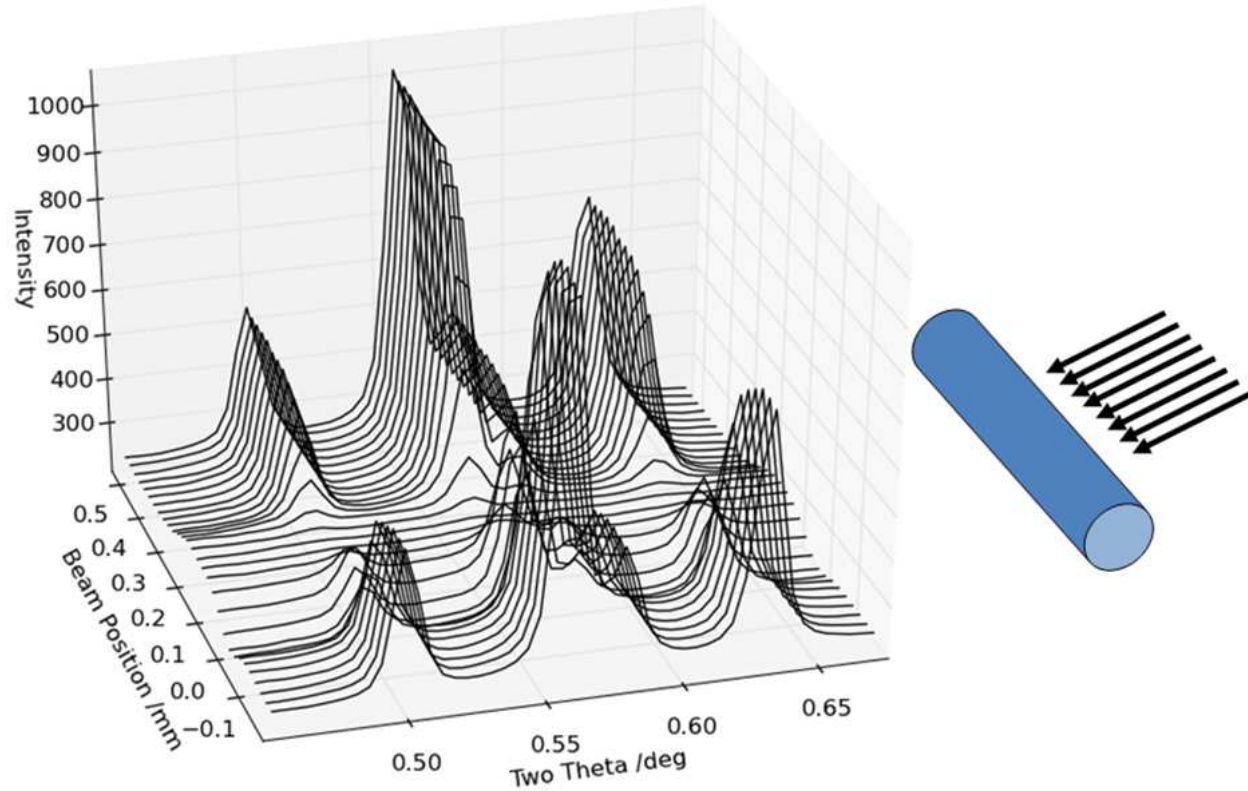

Fig. 6. Mapping along the length of the capillary after the radiation damage experiment 
On a log-scale the scale factor (which represents the diffracted intensity) gives an approximately linear behaviour. The LB sample begins with a significantly higher scale factor but then decays to be similar to the conventional sample.

There is a significant difference in the unit cell parameters. While the "a" parameter is almost equal for the two samples the "c" parameter is offset by around 0.05 Angstrom, or about $0.1 \%$, with the LB sample showing a larger unit cell parameter. For both of the samples the time-evolution of the cell parameters with radiation dose is rather similar

The peak width parameters start off with similar values and show a similar time evolution. During the early stages of radiation damage both samples show a rapid increase in peak widths. At long exposure times the peak widths continue to show a slow increase, but this is not observed for the unit cell parameters which stabilise. These results suggest two distinctly different time scales are present.

For the nano-template batch crystallised sample an extended data collection was performed overnight to investigate the long term radiation damage (900 images) and to find out whether crystalline diffraction peaks remained after extended exposure. A series of measurements were then performed along the length of the sample to measure the extent of the radiation damaged zone. Figure 6 shows the variation of the diffraction patterns as a function of position along the capillary. Very weak diffraction peaks were observed and the diffraction never completely disappeared (Fig. 4). Furthermore the zone of the radiation damage remains localised and "good" sample remained present on either side of the radiation damaged zone.

\section{Conclusion}

For powder diffraction experiments it was important to use the batch crystallization method in order to produce a large number of very small crystallites. Samples produced by the hanging drop method did not give good powder diffraction patterns. There was a small but significant improvement in the diffraction quality of the samples prepared with LB nano-template seeds as evidenced by a higher signal to background ratio. Upon exposure to the X-ray beam at room temperature both conventional and LB nano-templated samples showed a rapid initial damage phase and then a much slower second stage process. The damage rates were rather similar for both samples, but there was a significant difference in the length of the " $c$ " unit cell parameter, which was $0.1 \%$ larger for the nano-templated sample. Radiation damage was shown to be localized to the region where the beam hits the sample.

\section{Acknowledgement}

We are grateful to the ESRF for the provision of synchrotron beamtime allocation under project allocation CH2959.

\section{Author's Contributions}

JPW and CN designed the study. Samples were prepared by EP. The X-ray diffraction experiments were carried out by JPW, EP and CN. The data were analysed by JPW. The manuscript was written by JPW with contributions from $\mathrm{EP}$ and $\mathrm{CN}$.

\section{References}

Belmonte, L., E. Pechkova, S. Tripathi, D. Scudieri and C. Nicolini, 2012. Langmuir-blodgett nanotemplate and radiation resistance in protein crystals: State of the art. Crit. Rev. Eukaryotic Gene Exp., 22: 219-232. DOI: 10.1615/CritRevEukarGeneExpr.v22.i3.50

Finger, L.W., D.E. Cox and A.P. Jephcoat, 1994. A correction for powder diffraction peak asymmetry due to axial divergence. J. Applied Cryst., 27: 892-900. DOI: 10.1107/S0021889894004218

Hammersley, A.P., S.O. Svensson, M. Hanfland, A.N. Fitch and D. Hausermann, 1996. Twodimensional detector software: From real detector to idealised image or two-theta scan. High Pressure Res. Int. J., 14: 235-248. DOI: 10.1080/08957959608201408

Huang, T.C., H. Toraya, T.N. Blanton and Y. Wu, 1993. X-ray powder diffraction analysis of silver behenate, a possible low-angle diffraction standard. J. Applied Cryst., 26 : 180-184. DOI: 10.1107/S0021889892009762

Labiche, J.C., O. Mathon, S. Pascarelli, M.A. Newton and D.F. Carreiras et al., 2007. Invited article: The fast readout low noise camera as a versatile $\mathrm{x}$-ray detector for time resolved dispersive extended $x$-ray absorption fine structure and diffraction studies of dynamic problems in materials science, chemistry, and catalysis. Rev. Sci. Instrum., 78 091301- 091301. DOI: $10.1063 / 1.2783112$

Margiolaki, I. and J.P. Wright, 2008. Powder crystallography on macromolecules. Acta Cryst. Section Foundat. Cryst., 64: 169-180. DOI: 10.1107/S0108767307043735

Pechkova E., R. Gebhardt, C. Riekel, C. Nicolini, 2010. In Situ $\mu$ GISAXS: II. Thaumatin Crystal Growth Kinetic. Biophys. J., 99: 1256-1261. DOI: 10.1016/j.bpj.2010.03.068 
Pechkova, E. and C. Nicolini, 2001. Accelerated protein crystal growth by protein thin film template. J. Cryst. Growth, 231: 599-602. DOI: 10.1016/S0022-0248(01)01450-6

Pechkova, E., G. Tropiano, C. Riekel and C. Nicolini, 2004. Radiation stability of protein crystals grown by nanostructured templates: synchrotron microfocus analysis. Spectrochimica Acta Part B: Atomic Spectroscopy, 59: 1687-1693. DOI: 10.1016/j.sab.2004.07.020

Pechkova, E., S. Tripathi, R.B.G. Ravelli, S. McSweeney and C. Nicolini, 2009. Radiation stability of proteinase $\mathrm{K}$ crystals grown by LB nanotemplate method. J. Strucutarl Biol., 168: 409-418. DOI: 10.1016/j.jsb.2009.08.005
Vaughan, G.B.M., J.P. Wright, A. Bytchkov, M. Rossat and A. Snigirev et al., 2011. X-ray transfocators: focusing devices based on compound refractive lenses. J. Synchrotron Radiat., 18: 125-133. DOI: 10.1107/S0909049510044365

Von Dreele, R.B., 1999. Combined Rietveld and stereochemical restraint refinement of a protein crystal structure. J. Applied Cryst., 32: 1084 1089. DOI: $10.1107 / \mathrm{S} 002188989901064 \mathrm{X}$

Wright, J.P., 2004. Extraction and use of correlated integrated intensities with powder diffraction data. Zeitschrift Fur Kristallographie, 219: 791802. DOI: $10.1524 /$ zkri.219.12.791.55857 